\title{
Anneau vaginal à progestérone: évite les grossesses
}

Population Council

Follow this and additional works at: https://knowledgecommons.popcouncil.org/departments_sbsr-rh

Part of the Demography, Population, and Ecology Commons, Family, Life Course, and Society

Commons, International Public Health Commons, Maternal and Child Health Commons, and the Women's Health Commons

How does access to this work benefit you? Let us know!

\section{Recommended Citation}

"Anneau vaginal à progestérone: évite les grossesses," fact sheet. New York: Population Council, 2015.

This Fact Sheet is brought to you for free and open access by the Population Council. 


\section{ANNEAU VAGINAL À PROGESTÉRONE ÉVITE LES GROSSESSES}

\section{Les anneaux vaginaux sont une nouvelle ma- nière de fournir un moyen de contraception. L'anneau vaginal à progestérone (AVP) est un anneau vaginal contraceptif contenant de la progesterone.}

Il a été prouvé qu'il s'agit d'une méthode contraceptive sûre et efficace pour les femmes qui allaitent. Utilisé par les femmes qui allaitent, I'AVP évite les grossesses de diverses manières. En:

- inhibant la croissance folliculaire et, par conséquent, empêchant l'ovulation;

- renforçant les effets inhibiteurs de l'allaitement maternel sur l'ovulation;

- étendant l'aménorrhée post-partum;

- épaississant la glaire cervicale, et donc en empêchant les spermatozoïdes d'atteindre l'ovule.

\section{LA PREUVE}

L'efficacité de l'AVP pour éviter les grossesses a été démontrée dans trois domaines clés:

\section{PREVENTION DE L'OVULATION}

L'ovulation est régie par un système de communication complexe entre l'hypothalamus et l'hypophyse. Ce système de communication régule la production de deux hormones - I'hormone folliculostimulante (FSH) et l'hormone lutéinisante (LH) - essentielles pour ordonner aux ovaires de produire et libérer un ovule. Dans un cycle menstruel, l'effet de la FSH est la croissance des follicules de manière à accueillir un ovule et celui de la LH de déclencher la libération de l'ovule dans la trompe de Fallope. La LH émet aussi un signal ordonnant la production de progestérone et la préparation de l'endomètre pour une éventuelle grossesse. Le rôle de la progestérone est d'éviter le développement d'un nouveau follicule. Si l'ovule n'est pas fécondé, le niveau de progestérone chute après 14 jours, l'endomètre se détériore et le prochain saignement menstruel survient.

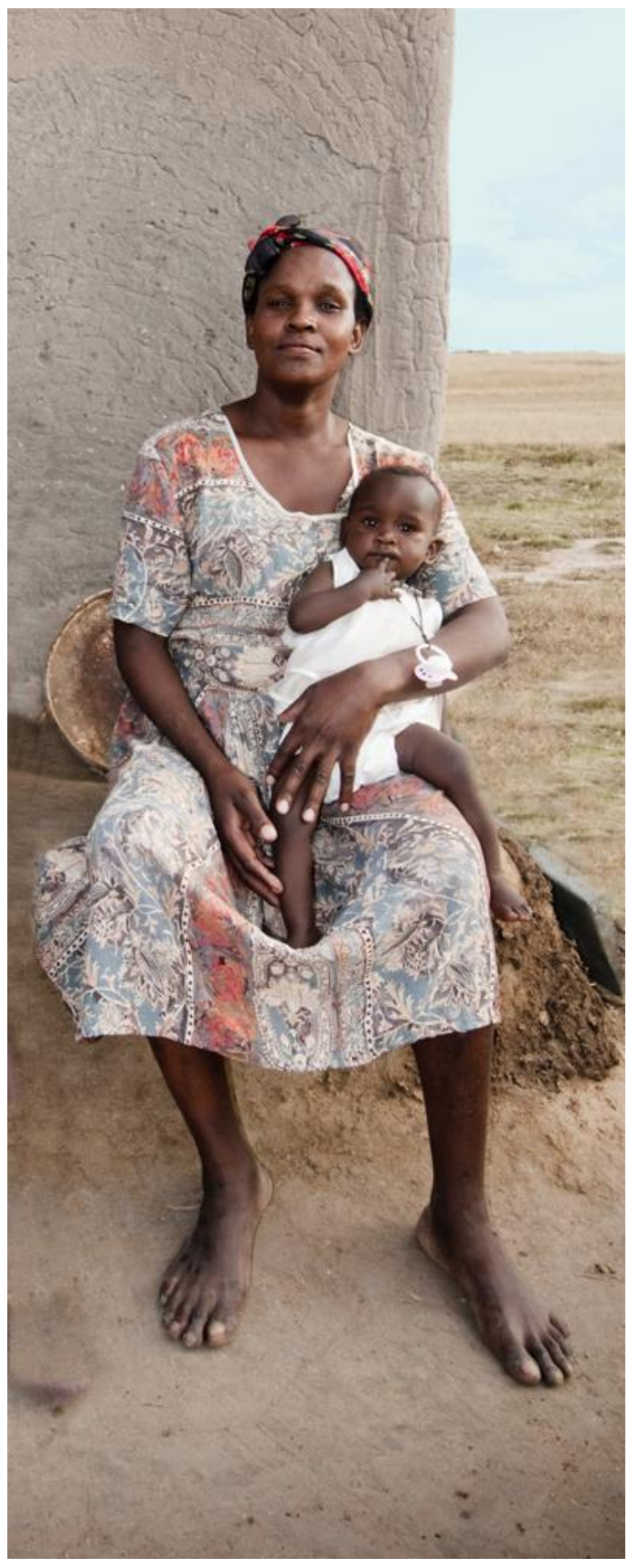




\section{L'AVP FONCTIONNE EN :}

- délivrant une faible quantité de progestérone agissant à la fois sur l'ovulation et la glaire cervicale. La progestérone présente dans l'anneau est diffusée dans les parois vaginales et ensuite dans la circulation sanguine.

- renforçant l'effet de la succion du bébé sur la production de l'hormone folliculostimulante (FSH) et de I'hormone lutéinisante (LH). L'effet de l'allaitement sur la réponse ovarienne à I'hypothalamus-hypophyse conduit à une suppression des FSH et $L H$. Ainsi, le niveau réduit en FSH et LH provoque un retard de l'ovulation.

- augmenter la réponse de la prolactine à la succion. La prolactine est l'hormone qui stimule et entretient la production de lait, et la progestérone augmente la production de prolactine. Les mères sont ainsi capables de nourrir leurs nourrissons plus longtemps qu'il n'aurait été autrement possible, et les plus longues durées d'allaitement prolongent la période d'aménorrhée post-partum.

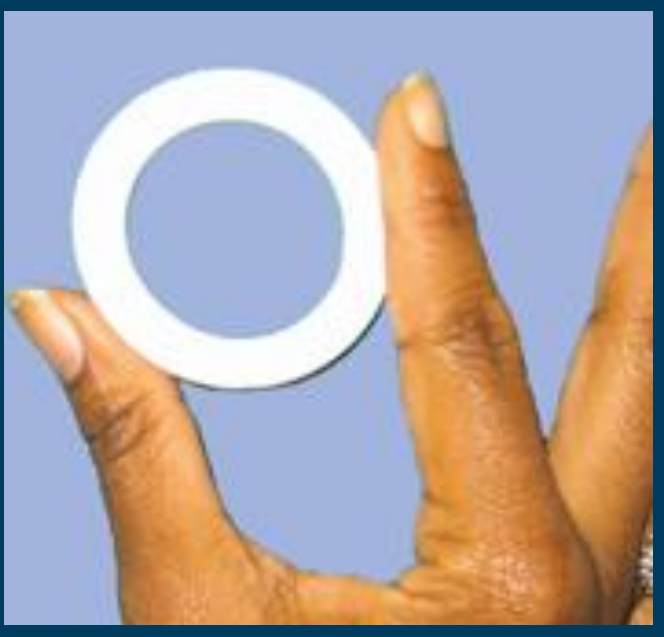

SOURCES: Diaz et al. 1991; McNeilly, Tay and Glasier 1994; Labbok 2008: Simpson-Herbert and Huffman 1981.

\section{RENFORCEMENT DE L'EFFET DE L'ALLAITEMENT SUR L'OVULATION}

L'allaitement joue également un rôle en retardant la reprise d'un cycle ovarien régulier après l'accouchement. La succion effectuée par le nourrisson sur le sein maternel supprime la production de FSH et de LH nécessaires à l'ovulation (McNeilly, Tay and Glasier, 1994; McNeilly, 1997). Nous savons que la succion déclenche une réaction en chaine de signaux entre l'hypothalamus, I'hypophyse et les ovaires, régulant ainsi l'ovulation. En grandissant, le bébé tète moins et la réponse à la succion s'amenuise. Quand la réponse à la succion se réduit, les FSH et les LH retrouvent leurs niveaux et leurs processus de signalisation normaux, les follicules durables se développent et l'ovulation est déclenchée.

\section{EXTENSION DE L'AMÉNORRHÉE DUE À LA LACTATION}

L'aménorrhée due à la lactation est la période d'infertilité naturelle suivant immédiatement l'accouchement. La durée de l'aménorrhée d'une femme dépend de la durée et de la fréquence de l'allaitement. La durée de l'allaitement dépend d'un certain nombre de facteurs, dont la capacité de la mère à allaiter, son désir de le faire, et le moment de l'introduction des aliments de sevrage. Lors de tests comparatifs des deux méthodes, des taux d'aménorrhée plus élevés ont été observés chez les utilisatrices d'AVP que chez celles qui ont choisi les DIU; en moyenne, l'aménorrhée est plus longue de 5 mois (cité dans Massai et al. 2000).

\section{MULTIPLIER LES OPTIONS CONTRACEPTIVES}

En 2015, I'AVP a été ajouté à la ligne directrice de l'utilisation de contraceptifs de la Liste des Médicaments Essentiels (LME) et Critères d'Éligibilité Médicale (CEM) de l'Organisation Mondiale de la Santé (OMS) destinée aux fournisseurs.

\section{SOMMAIRE}

L'AVP étend la gamme de contraceptifs à la disposition des femmes qui allaitent. II a été prouvé qu'il s'agit d'une méthode sûre et efficace. L'AVP a le pouvoir de contribuer à l'amélioration de la santé de la maman et de son bébé.

\section{Références}

Díaz, Soledad, P. Miranda, A. Brandeis, H. Cárdenas, and Horacio B. Croxatto. 1991. "Mechanism of action of progesterone as contraceptive for lactating women. In Frontiers in Human Reproduction Part II": Contraception and Sterilization. Eds. M. Seppala and L. Hamberger, Annals, New York Academy of Sciences, pp: 11-21.

Labbok, M. 2008. "Breastfeeding, fertility and family planning." In J. Sciarra (ed.). Fertility Regulation, Psychosomatic Problems, and Human Sexuality. The Global Library of Women's Medicine. DOI: 10.3843/GLOWM.10397

McNeilly, Alan S. 1997. “Lactation and fertility” Journal of Mammary Gland Biology and Neoplasia 2(3): 291-298.

McNeilly, Alan S., Clem C.K. Tay, and Anna Glasier. 1994. "Physiological mechanisms underlying lactational amenorrhea" Annals of the New York Academy of Sciences 709: 145-155.

Massai, Rebeca, Soledad Diaz, Ted Jacaanicz, and Horacio B. Croxatto. 2000. "Vaginal rings for contraception in lactating women" Steroids 65: 703-707.

Simpson-Herbert, Maylin and Sandra L. Huffman. 1981. "The contraceptive effect of breastfeeding" Studies in Family Planning 12(4):125-133.

POUR PLUS D'INFORMATIONS

Pour plus d'informations concernant le Conseil, veuillez consulter notre site web www.popcouncil.org 